\title{
Posterior Circulation Infarcts and Carotid Disease. Is There a Correlation?
}

\author{
Ingram $O D^{1 *}$, Ali $T^{2}$, Khosla $N^{1}$, Burney $K^{1}$ and Kakar $P^{1}$ \\ ${ }^{1}$ Epsom and St Helier University Hospitals NHS Trust, UK \\ ${ }^{2}$ Ashford and St Peter's Hospitals NHS Foundation Trust, UK
}

*Corresponding author: Owen Ingram, MA, MBBS, MRCP, ST4 Geriatric Medicine, Epsom and St Helier University Hospitals NHS Trust, UK, Tel: 07763557055

\begin{abstract}
We present a case of a 66-year-old man who presented with a history of Right Hemianopia and Right sided facial droop and weakness. CT and MRI brain imaging demonstrated areas of infarction affecting the vascular regions supplied by Left Middle Cerebral Artery and Left Posterior Cerebral Artery circulation. Carotid Duplex Arterial imaging demonstrated moderate Left Internal Carotid Artery Stenosis and CT Angiography demonstrated a dominant Left Posterior Communicating Artery. The origin of the multi-territory Stroke as demonstrated on the MRI scan was his underlying Carotid disease. The patient underwent a successful Left Carotid Endarterectomy.

This case highlights the fact that patients presenting with isolated Posterior Cerebral Arteryterritory stroke or mixed Posterior Cerebral Artery and Middle Cerebral Artery territory strokes and ipsilateral Internal Carotid Artery stenosis, should have a low threshold to be assessed with CT or MR Brain Angiography to determine if variants in Cerebral Circulation exist.
\end{abstract}

\section{Keywords}

Ischaemic stroke, Carotid artery stenosis, Foetal variant posterior, Communicating artery

\section{Introduction}

Stroke is a common condition with multiple underlying aetiologies. Multi-territory Stroke involving both Anterior and Posterior Cerebral circulation is normally attributed to Cardio-embolic disease. In a small but significant proportion of the adult population there is a Foetal variant Posterior Communicating Artery [PCOM] which supplies the Posterior Cerebral Artery [PCA] from the Anterior Cerebral circulation [1]. In these patients symptomatic ipsilateral Carotid Artery disease can pres- ent with both Anterior Cerebral circulation and PCA territory Stroke [2].

\section{Case Report}

A 66-year-old man presented with a one day history of Right Hemianopia and a 6 hour history of Right sided facial droop and weakness. His risk factors included Hypertension, Hypercholesterolaemia and a 50 pack year smoking history. Clinical examination confirmed above findings and initial Brain Computed Tomography [CT] scan demonstrated areas of low attenuation in the left Cerebral Hemisphere, involving the left Caudate Nucle-

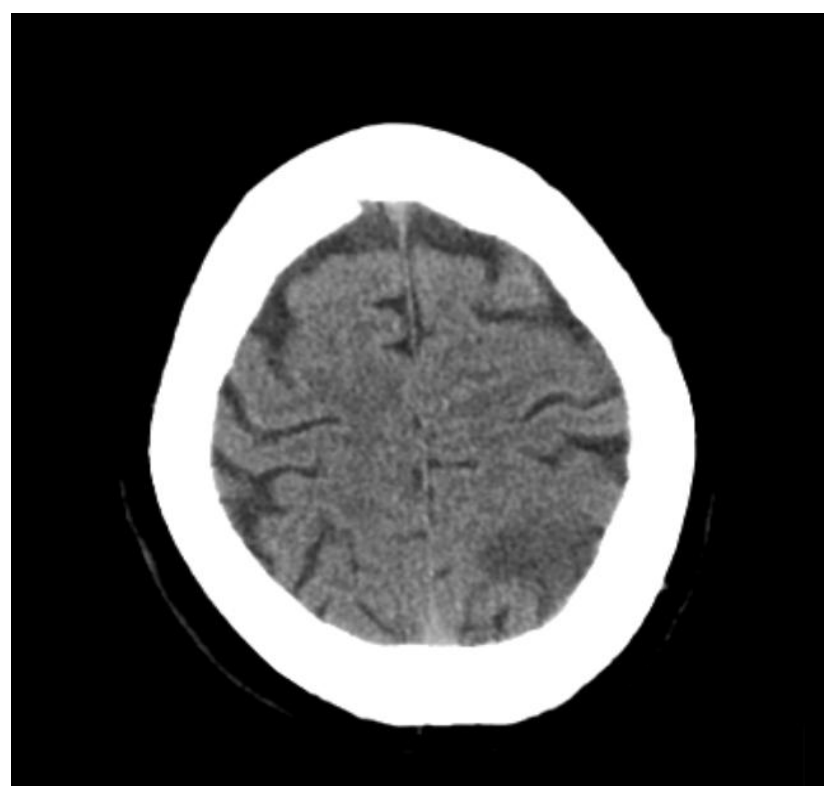

Figure 1: CT Brain scan demonstrating low attenuations in the Left Occipital Lobe and Left Parietal Lobe posteriorly.

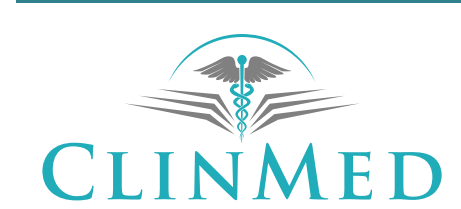

INTERNATIONAL LIBRARY
Citation: Ingram OD, Ali T, Khosla N, Burney K, Kakar P (2019) Posterior Circulation Infarcts and Carotid Disease. Is There a Correlation?. Int J Neurol Neurother 6:088. doi.org/10.23937/2378-3001/1410088 Accepted: August 12, 2019: Published: August 14, 2019

Copyright: (c) 2019 Ingram OD, et al. This is an open-access article distributed under the terms of the Creative Commons Attribution License, which permits unrestricted use, distribution, and reproduction in any medium, provided the original author and source are credited. 


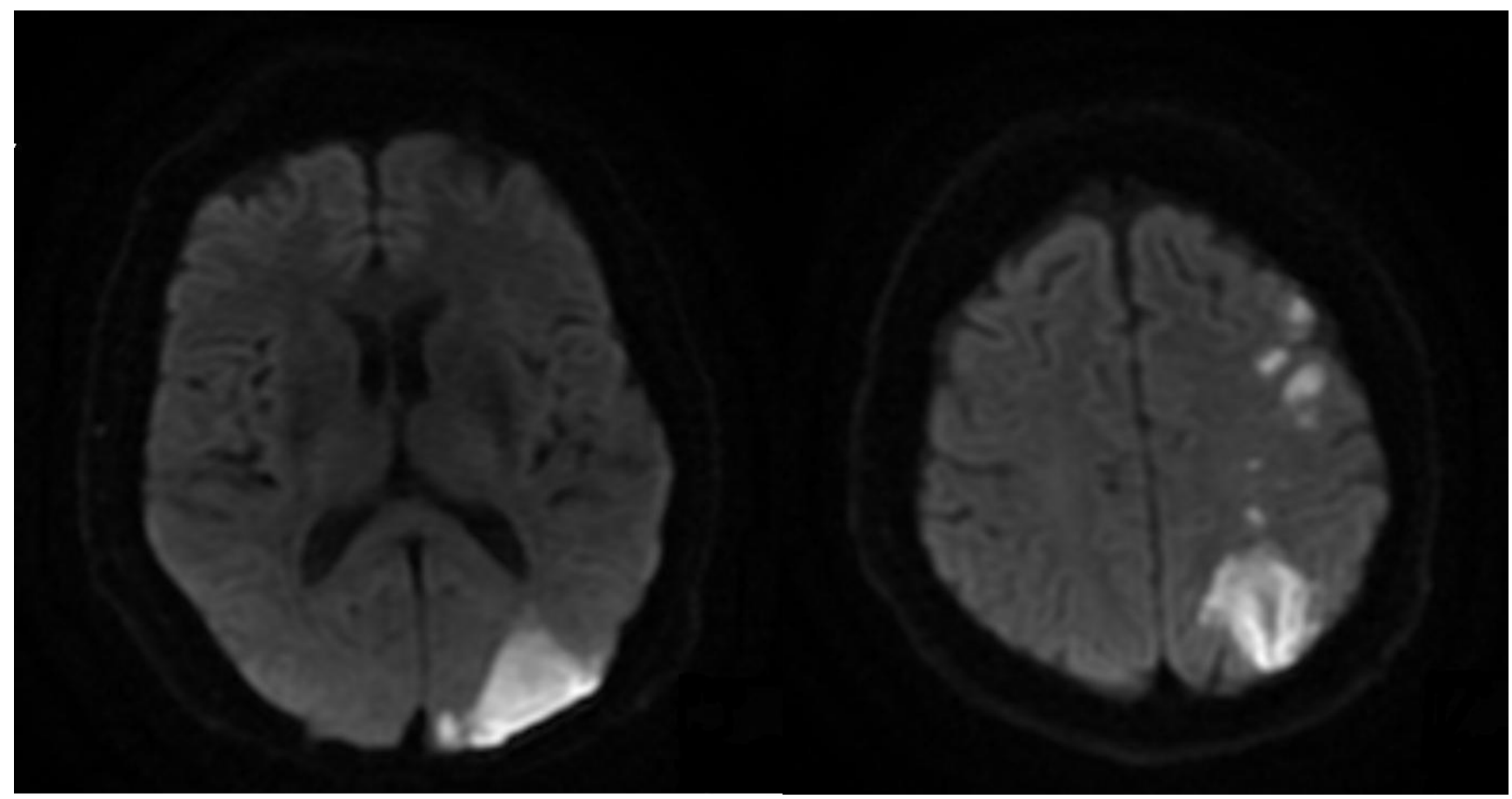

Figure 2: MRI Diffusion Weighted Imaging of the Brain demonstrating multiple areas of infarction affecting the vascular regions supplied by Left MCA and Left PCA circulation.

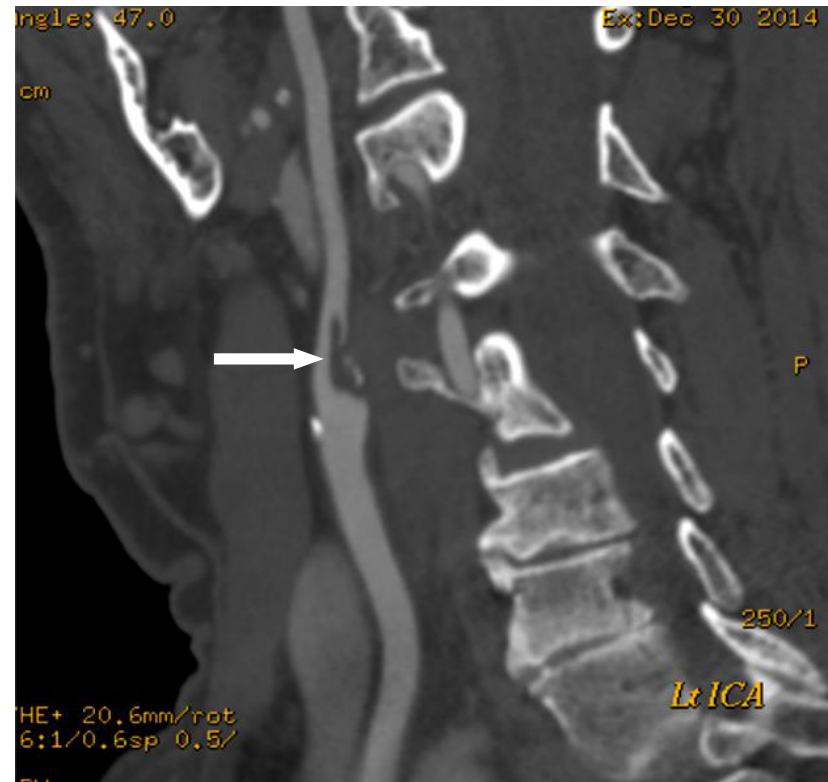

Figure 3: CT Carotid Angiography demonstrating a large ruptured plaque in the Left Internal Carotid Artery (white arrow).

us, left Occipital Lobe and left Parietal Lobe posteriorly (Figure 1). Brain Magnetic Resonance Imaging [MRI] confirmed CT findings and demonstrated multiple areas of infarction affecting the vascular regions supplied by Left Middle Cerebral Artery [MCA] and Left PCA circulation (Figure 2). The suggestion that these Infarcts were more than likely to represent a Cardio-Embolic source was mooted and appropriate Cardiac investigations were instituted.

Echocardiography proved to be normal with no evidence of a structural or embolic Cardiac source and
Cardiac monitoring demonstrated Sinus Rhythm without any significant Atrial Arrhythmias. Haematological and Biochemical parameters were within normal limits. Carotid Duplex Arterial imaging demonstrated patent bilateral Common Carotid Arteries with a moderate Left Internal Carotid Artery [ICA] Stenosis quantified through velocity measurements at $50-59 \%$. This was initially thought to be a coincidental finding as Posterior Circulation Infarcts could not be normally explained with Carotid Stenosis; however it was decided to qualify these findings further with CT Angiography.

CT Angiography demonstrated a large ruptured plaque of heterogeneous morphology in the Left ICA associated with a propagating Thrombus (Figure 3 ). These findings were associated with a dominant Left PCOM feeding the Left Posterior Cerebral circulation and thereby explaining the fact that the plaque present in the Left ICA was able to seed the Left Middle Cerebral and the Left Posterior Cerebral circulation and in fact; the origin of the multi-territory Stroke as demonstrated on the MRI scan was not Cardio-Embolic but in fact related to his underlying Carotid disease. The patient underwent a successful Left Carotid Endarterectomy and has made an excellent functional recovery. He remains on high dose Statin and Anti-Platelet therapy. The decision to investigate the presumed moderate Carotid Stenosis demonstrated on Duplex imaging with CT Angiography was central to the diagnosis and further management.

\section{Discussion}

The importance of this case is to highlight unusual presentations of common conditions. Stroke has multiple aetiologies including Atherosclerotic Large Vessel 
Extra-Cranial disease and Cardio-Embolic disease accounting for nearly $50 \%$ of all Strokes. The Carotid vascular tree generally supplies the Middle and Anterior Cerebral circulation and the Vertebro-Basilar system supplies the Posterior Cerebral circulation. Where Imaging suggests multi-territory [unilateral and bilateral] infarcts especially involving Anterior and Posterior circulation, the diagnostic balance shifts in favour of a Cardio-Embolic source as it is a commonly held view that Carotid disease related Strokes should not involve the Posterior circulation territories. While this view is accurate in the majority of cases, a high threshold of suspicion must be exercised. In a small but significant proportion of the adult population, there is a Foetal variant PCOM and in these individuals, it is the PCOM [arising from the MCA and thereby Anterior circulation] that provides the majority of blood supply to the Posterior Cerebral areas.

Foetal variant PCOM is the commonest anatomical variant of the Posterior circulation of the Circle of Willis. It is related to hypoplasia or absence of the $\mathrm{P} 1$ segment of the PCA. It occurs in up to $29 \%$ of the adult population and is more commonly found unilaterally [1]. CT Angiography has been shown to be both a sensitive and specific tool in identifying Foetal variant PCOM [3]. It must be noted that the Foetal variant PCOM does not per se increase the incidence of PCA territory ischaemic Stroke [4]. However, in patients with Anterior and Posterior Circulation territory ischaemic Stroke with ipsilateral Carotid disease, consideration must always be given to further imaging to determine if the Foetal variant PCOM exists [2].

Our case demonstrates symptomatic Carotid Artery Disease resulting in both MCA and PCA territory strokes due to a Foetal variant PCOM. Reliance on Carotid Du- plex imaging alone does not allow for Intra Cerebral Vasculature to be determined and there is a risk that patients with the above presentation with mild to moderate ipsilateral Carotid Stenosis may be offered best medical treatment instead of Surgery based on the presumption that the Stroke may be Cardio-embolic in nature. Given the benefits of Carotid Endarterectomy in symptomatic stenosis [5] and the incidence of Foetal variant PCOM; we highlight the fact that patients presenting with isolated PCA territory stroke or mixed PCA and MCA territory strokes and ipsilateral ICA stenosis, should have a low threshold to be assessed with CT or MR Brain Angiography to determine if variants in Cerebral Circulation exist.

\section{References}

1. Van Raamt AF, Mali WP, van Laar PJ, van der GraafY (2006) The Fetal Variant of the Circle of Willis and Its Influence on the Cerebral Collateral Circulation. Cerebrovasc Dis 22: 217-224.

2. Yang JH, Choi HY, Nam HS, Kim SH, Han SW, et al. (2007) Mechanism of infarction involving ipsilateral carotid and posterior cerebral artery territories. Cerebrovasc Dis 24: 445-451.

3. Van der Lugt A, Butler TC, Govaere F, Siepman DA, Tanghe HL, et al. (2004) Accuracy of CT angiography in the assessment of the fetal origin of the PCOM. Eur Radiol 14: 1627-1633.

4. De Monye C, Dippel DW, Siepman TA, Dijkshoorn ML, Tanghe HL, et al. (2008) Is a fetal origin of the posterior cerebral artery a risk factor for TIA or ischemic stroke? A study with 16-multidetector-row CT angiography. J Neurol 255: $239-245$.

5. NASCET, Barnett HJM, Taylor DW, Haynes RB, Sackett DL, et al. (1991) Beneficial Effect of Carotid Endarterectomy in Symptomatic Patients with High-Grade Carotid Stenosis. N Engl J Med 325: 445-453. 